\title{
Antisense oligonucleotides against monoacylglycerol acyltransferase 1 (Mogat1) improve glucose metabolism independently of Mogat1
}

Andrew J. Lutkewitte, Jason M. Singer, Trevor M. Shew, Michael R. Martino,

Angela M. Hall, and Brian N. Finck*

Center for Human Nutrition, Washington University School of Medicine, St. Louis, MO

Running title: Mogat1 ASO treatment improves glucose metabolism independent of Mogat1

*Acceptance of proofs and correspondence to:

Brian N. Finck

bfinck@wustl.edu

Center for Human Nutrition,

Washington University School of Medicine, 660 Euclid Ave.,

Box 8031,

St. Louis, MO 63110, United States of America.

Declarations of interest: none 


\section{ABSTRACT}

Objective: Monoacylglycerol acyltransferase (MGAT) enzymes catalyze the synthesis of diacylglycerol from monoacylglycerol. Previous work has suggested the importance of MGAT activity in the development of obesity-related hepatic insulin resistance. Indeed, antisense oligonucleotide (ASO)mediated knockdown of the gene encoding MGAT1, Mogat1, reduced hepatic MGAT activity and improved glucose tolerance and insulin resistance in high fat diet (HFD) fed mice. However, recent work has suggested that some ASOs may have off-target effects on body weight and metabolic parameters via activation of the interferon alpha/beta receptor 1 (IFNAR-1) pathway.

Methods: Mice with whole-body Mogat1 knockout or a floxed allele for Mogat1 to allow for liverspecific Mogat1-knockout (by either a liver-specific transgenic or adeno-associated virus-driven Cre recombinase) were generated. These mice were placed on a high fat diet and glucose metabolism and insulin sensitivity was assessed after 16 weeks on diet. In some experiments, mice were treated with control or Mogat1 or control ASOs in the presence or absence of IFNAR-1 neutralizing antibody.

Results: Genetic deletion of hepatic Mogat1, either acutely or chronically, did not improve hepatic steatosis, glucose tolerance, or insulin sensitivity in HFD-fed mice. Furthermore, constitutive Mogat1 knockout in all tissues actually exacerbated HFD-induced weight gain, insulin resistance, and glucose intolerance on a HFD. Despite markedly reduced Mogat1 expression, liver MGAT activity was unaffected in all knockout mouse models. Mogat1 overexpression hepatocytes increased liver MGAT activity and TAG content in low-fat fed mice, but did not cause insulin resistance. Interestingly, Mogat1 ASO treatment improved glucose tolerance in both wild-type and Mogat1 null mice, suggesting an off target effect. Inhibition of IFNAR-1 did not block the effect of Mogat1 ASO on glucose homeostasis. Conclusion: These results indicate that genetic loss of Mogat1 does not affect hepatic MGAT activity or metabolic homeostasis on HFD and show that Mogat1 ASOs improve glucose metabolism through effects independent of targeting Mogat1 or activation of IFNAR-1 signaling. 
Key words: monoacylglycerol acyltransferase, insulin resistance, antisense oligonucleotides, interferon receptor

\section{INTRODUCTION}

Nonalcoholic fatty liver disease (NAFLD) results from ectopic intrahepatic lipid accumulation (steatosis) and is believed to be a key driver of many metabolic abnormalities associated with obesity including insulin resistance and diabetes [1,2]. In obesity, the liver is overloaded with fatty acids from dietary intake, increased adipose tissue lipolysis, and higher rates of de novo lipid synthesis [3-5]. The liver must efficiently store these lipids as neutral triacylglycerol (TAG) to prevent deleterious effects of toxic lipid intermediates such as activation of inflammation, ER stress, and insulin resistance [6].

There are two pathways of TAG synthesis in liver: the glycerol-3-phosphate (G-3-P) pathway and the monoacylglycerol O-acyltransferase (MGAT) pathway. These pathways converge at diacylglycerol (DAG), which is the sole precursor of TAG [7-9]. MGATs acylate monoacylglycerol to form DAG and this enzymatic activity is encoded by several genes including Mogat1, Mogat2, and Dgat1 in mice [10-13]. The importance of MGAT activity in dietary fat absorption by intestinal enterocytes has long been studied $[8,14]$. In contrast, the G-3-P pathway is considered to be the primary route of TAG synthesis in tissues other than the intestine and the MGAT pathway is believed to be an auxiliary pathway. We have recently demonstrated that Mogat1 is also highly expressed in adipocytes and may function to suppress aberrant lipolysis [15]. In addition, the expression and activity of the MGATs are increased in humans with NAFLD and mouse models with hepatic steatosis [16-20]. Suppression of hepatic and adipose tissue Mogat1 expression through use of antisense oligonucleotides (ASO) reduces hepatic MGAT activity and improves hepatic insulin sensitivity and glucose metabolism without reducing hepatic steatosis in obese mice $[17,18]$. 
Second generation ASOs are known to target multiple tissues including liver, adipose tissue, and intestine, all of which have MGAT activity [21]. Moreover, off-target effects of ASO treatment have also been described [22]. McCabe and colleagues recently demonstrated an improvement in adipose tissue metabolism by ASO targeting TTC39B mRNA that was independent of target gene knockdown. These effects were mediated through the activation of interferon alpha/beta receptor 1 (IFNAR-1) signaling in adipose-derived macrophages [22]. Thus, we sought to obtain rigorous and independent confirmation that hepatic Mogat1 plays an important role in obesity-related hepatic insulin resistance and metabolic abnormalities by using novel liver-specific and global Mogat1 knockout mice. Our data surprisingly suggest that Mogat1 ASO treatment improves glucose metabolism through Mogat1-independent mechanisms and that the effects are not mediated through IFNAR-1 activation.

\section{METHODS}

\subsection{Generation of mouse models}

All mouse studies were approved by the Institutional Animal Care and Use Committee of Washington University. Due to the resistance of female mice to the effects of high fat diet, male mice in the C57BL6/J background were used in all studies. Mice were group housed and maintained on standard laboratory chow on a $12 \mathrm{~h}$ light/dark cycle. At eight weeks of age mice were placed on control low-fat diet (LFD, Research Diets, $10 \mathrm{kcal} \%$ fat matched sucrose, D12450J) or high-fat diet (HFD, Research Diets, $60 \mathrm{kcal} \%$ fat, D12492) for the durations indicated. ASO treatments were performed as previously described [17]. Briefly, starting at 16 weeks on diet, mice were given twice weekly intraperitoneal injections of ASO directed against Mogat1 (sequence 1: 5'-GATCTTGGCCACGTGGAGAT-3' (20-mer) or sequence 2: 5'-TGGCCACGTGGAGATACGAT-3" (20-mer) where indicated) or scramble control (Ionis, Pharmaceuticals, Inc., Carlsbad, CA; 25 mg/kg body weight) for three weeks. 
Embryonic stem cells used to generate Mogat1 whole-body knockout and Mogat1 floxed mice were obtained from the Knockout Mouse Consortium (project\# CSD35789) and mice harboring this floxed allele have been previously described [15]. Mogat1 floxed mice were crossed with mice expressing the Cre recombinase transgene under the control of the albumin promoter (Jackson Laboratory, B6.Cg-Speer6-ps1Tg (Alb-cre) 21Mgn/J). Acute liver-specific knockout mice were generated by retro-orbital injection of Mogat1 floxed mice with $2.0 \times 10^{11}$ genomic copies (GC) of adeno-associated virus serotype 8 (AAV8) expressing Cre recombinase under the control of human thyroid hormonebinding globulin (TBG) promoter (Vector Biolabs, VB1724). Control mice received AAV8 expressing enhanced green fluorescent protein (GFP) under control of the same promoter (Vector Biolabs, VB1743). For shRNA studies, C57BL6/J mice (Jackson Laboratory) received retro-orbital injection of either control AAV8-GFP-U6-scramble-shRNA (Vector Biolabs) or AAV8-GFP-U6-Mogat1-shRNA (sequences 5-UUUCACCCUCAUGGAAUAUUCGUGCCU-3 and 5-CAAGACGCAAUGUAUGAUUCAAUGGGA-3 [20]; pooled (2.0 $\times 10^{11} \mathrm{GC}$ total) before injection (Vector Biolabs). For hepatic Mogat1 overexpression, eight-week-old male C57BL6/J mice were given LFD or HFD for six weeks, then administered AAV8-TBGeGFP or AAV8-TBG-mouse-Mogat1 by retro-orbital injection (Vector Biolabs, VB1743, custom refseq\# BC106135).

In the IFNAR-1 inhibition study, HFD-fed male C57BL/6J mice were obtained from Jackson Laboratory after 12-weeks of diet. After acclimation and four additional weeks of HFD feeding, mice were weight matched into four treatment groups. Mice were given scramble ASO or ASOs targeting Mogat1 (5'-TGGCCACGTGGAGATACGAT-3" (20-mer)) as described above. During each ASO treatment mice were also given IP injections of IgG control (BioCell InVivolMAb, \#BE0083, clone MOPC-21) or a monoclonal neutralizing antibody targeting interferon alpha/beta receptor 1 (IFNAR-1) (BioCell InVivo/MAb, \#BE0241, clone MAR1-5A3) [23]. Antibody treatments were given as IP injections of 250 ug per mouse in 100 uL of dilution buffer (BioCell, InVivoPure Ph 6.5 Dilution Buffer, \#IP0065) twice a week 
for the first two weeks of ASO treatment followed by three separate injections of 500 ug per mouse the third week of ASO treatments.

Prior to sacrifice mice were fasted for 4 hours starting at 0900. Mice were euthanized via $\mathrm{CO}_{2}$ asphyxiation. Blood was collected from venipuncture of the inferior vena cava into EDTA-coated tubes and plasma was removed by centrifugation. Liver and other tissues were immediately collected, flashfrozen in liquid nitrogen, and stored at $-80^{\circ} \mathrm{C}$ until further use.

\subsection{Metabolic phenotyping of mouse models.}

Glucose tolerance tests were performed in mice fasted for 5 hours starting at 0900. Mice were given an intraperitoneal (IP) injection of glucose ( $1 \mathrm{~g} / \mathrm{kg}$ body weight dissolved in saline) and blood glucose was measured from the tail using a One Touch Ultra glucometer (Life Scan Inc.) at times indicated. For insulin tolerance tests, mice were fasted for 4 hours before IP injections of recombinant Humulin $\mathrm{R}^{\circledast}(0.75 \mathrm{U} / \mathrm{kg}$ body weight in saline). Tolerance tests were performed one week apart to allow the mice to recover starting at 15 and 16 weeks of diet or week two and three of acute treatments. Body composition was determined in fed mice using ECHO MRI. Because the whole-body null mice had differences in body weights compared to WT mice, these mice were dosed based on lean mass (1 g glucose/kg lean mass and $1.5 \mathrm{U}$ insulin/kg lean mass).

\subsection{Liver lipids and plasma metabolites.}

Frozen liver pieces were homogenized with bead disruption in PBS (100 mg ml). Lipids were solubilized in $1 \%$ sodium deoxycholate via vortexing and heating at $37^{\circ} \mathrm{C}$ for $5 \mathrm{~min}$. Triglycerides were measured enzymatically using the Infinity Triglyceride colorimetric assay kit (Thermo Fisher, TR22421) and normalized to mg tissue (wet weight). Plasma insulin was determined by Singulex Immunoassay (Millipore Sigma) via the Washington University Core Laboratory for Clinical Studies.

\section{4 mRNA isolation and quantitative PCR.}


Total liver RNA was isolated from frozen liver samples using RNA STAT (Iso Tech, CS-502) according to the manufacturer's protocol. RNA was reverse transcribed into cDNA using Taqman high capacity reverse transcriptase (Life Technologies, 43038228). Quantitative PCR was performed using Power SYBR green (Applied Biosystems, 4367659) and measured on an ABI PRISM 7500 or ABI QuantStudio 3 sequence detection system (Applied Biosystems). Results were quantified using the $2^{-\Delta \Delta c t}$ method and shown as arbitrary units relative to control groups. Primer sequences are listed in supplemental table 1.

\subsection{Glycogen concentration analyses}

Samples of frozen tissues (30 to $90 \mathrm{mg}$ ) were hydrolyzed in $0.3 \mathrm{ml}$ of $30 \%$ (wt/vol) $\mathrm{KOH}$ solution in a boiling water bath for $30 \mathrm{~min}$. At 10 and $20 \mathrm{~min}$ of the incubation, tubes were vortexed to facilitate digestion. After cooling to room temperature, $0.1 \mathrm{ml}$ of $1 \mathrm{M} \mathrm{Na} 2 \mathrm{SO} 4$ and $0.8 \mathrm{ml}$ of ethanol were added, the samples were then boiled again for $5 \mathrm{~min}$ to facilitate precipitation of glycogen and then centrifuged at $10,000 \times \mathrm{g}$ for $5 \mathrm{~min}$. The glycogen pellet was dissolved in $0.2 \mathrm{ml}$ of water, and two additional ethanol precipitations were performed. The final pellet was dried and dissolved in $0.2 \mathrm{ml}$ of $0.3 \mathrm{mg} / \mathrm{ml}$ amyloglucosidase (Sigma Aldrich 11202332001) in $0.2 \mathrm{M}$ sodium acetate buffer (pH 4.8) and incubated for $3 \mathrm{~h}$ at $40^{\circ} \mathrm{C}$. The reaction mixture was diluted two- to fivefold with water. To determine the glucose concentration, $5 \mathrm{ml}$ of the diluted sample was added to $0.2 \mathrm{ml}$ of the glucose assay solution $(0.3 \mathrm{M}$ triethanolamine-KOH (pH 7.5), 1 mM ATP (Sigma Aldrich, A6559), 0.9 mM b-NADP (Sigma Aldrich, 10128031001), and $5 \mathrm{mg} / \mathrm{ml}$ of G-6P dehydrogenase (Sigma Aldrich, 10165875001)). The absorbance at $340 \mathrm{~nm}$ was determined before and after addition of $1 \mathrm{mg}$ of hexokinase (Sigma Aldrich, 11426362001). Glycogen content is expressed as micromoles of glucosyl units per gram (wet weight).

\subsection{Primary hepatocyte isolations.}

Primary hepatocytes were isolated as previously described [24]. Briefly, 10-16-week-old female mice were given an overdose of isoflurane prior to perfusions. The livers were perfused via 
catheterization of the hepatic portal vein and flushed with $30 \mathrm{ml}$ of $\mathrm{HBSS}\left(\mathrm{Ca}^{2+} / \mathrm{Mg}^{2+}\right.$-free, $\left.0.5 \mathrm{mM} \mathrm{EGTA}\right)$ prior to digestion with $20 \mathrm{ml}$ of collagenase solution (Type IV collagenase (sigma S5138) at $1 \mathrm{mg} / \mathrm{ml}$ in DMEM (serum free, $1 \mathrm{mM}$ sodium pyruvate). Following perfusion, the livers were removed and disrupted in the collagenase solution. Cells were added to ice-cold complete DMEM (10\% FBS, 1 mM sodium pyruvate, $100 \mathrm{U}$ PenStrep, $0.25 \mu \mathrm{g} / \mathrm{ml}$ amphotericin b) and passed through a $50 \mu \mathrm{M}$ filter prior to centrifugation at $50 \mathrm{~g} \times 2 \mathrm{~min}$. The supernatant was removed and hepatocytes were resuspended in complete DMEM and washed two more times. 500,000 cells were then directly added to RNA STAT for RNA isolation and quantification.

\subsection{MGAT enzymatic assay.}

MGAT activity was determined as previously described [17]. Liver $(50 \mathrm{mg} / \mathrm{ml})$ was homogenized by probe sonication in ice-cold membrane buffer (50 mM Tris-HCl pH 7.4, 1 mM EDTA, 250 mM sucrose, and complete protease inhibitors table (Roche Diagnostics, A32965)). Homogenates were clear of whole-cell debris by low speed centrifugation at $500 \mathrm{~g} \times 10 \mathrm{~min}$ at $4^{\circ} \mathrm{C}$. The resulting supernatants were spun at $100,000 \mathrm{~g} \times 60 \mathrm{~min}$ at $4^{\circ} \mathrm{C}$ using a Beckman benchtop ultra-centrifuge. The cytosolic fractions (supernatant) were removed and the membranes were reconstituted via pipetting in membrane buffer without protease inhibitors. Proteins were quantified using the bicinchoninic acid (BCA) assay according to the manufacture's protocols (Themo Fisher, 23225). Fifty micrograms of membrane were incubated in assay buffer ( $5 \mathrm{mM} \mathrm{MgCl} 2,1.25 \mathrm{mg} / \mathrm{ml} \mathrm{BSA}, 200 \mathrm{mM}$ sucrose, $100 \mathrm{mM}$ Tris-HCl (pH 7.4)) containing $20 \mu \mathrm{M}\left[{ }^{14} \mathrm{C}\right]$ oleoyl-CoA (American Radiolabeled Chemicals, ARC 0527), and $200 \mu \mathrm{M}$ sn-2-oleoylglycerol (Caymen Chemical, 16537) for $10 \mathrm{~min}$. The reaction was stopped with $50 \mu \mathrm{l}$ 1\% phosphoric acid. Lipids were extracted in 2:1 v/v \% CHCl3:MeOH and separated by thin-layer chromatography in hexane/ethyl ether/acetic acid (80:20:1, v /v/v \%). Samples were run against standards for oleic acid, 1,3 diacylglycerol and triglyceride and corresponding spots were scraped from the plate and ${ }^{14} \mathrm{C}$ radioactivity was measured via scintillation counter. Backgrounds were calculated from reaction 
mixtures without membrane fractions.

\subsection{Western blotting.}

Membrane and cytosolic protein samples were isolated as described in (Section 2.7). Fifty micrograms of protein were loaded onto 4-15\% acrylamide pre-cast gels (BioRad, 64329760) in Lamellae loading buffer (BioRad, 161-0737). Proteins were transferred to PVDF membranes in Tris-glycine buffer with $10 \%$ methanol. Following transfer, membranes were blocked in 5\% BSA in TBS for 1 hour prior to overnight antibody incubations in 5\% BSA in TBS. Antibodies used are as follows: Mogat1 (Santa Cruz Biotechnologies, 32387, 1:500) green fluorescent protein (GFP, Cell Signaling Technologies, 2555, 1:1000), Calnexin (Enzo Life Sciences, ADI-SPA-860, 1:1000) and mouse $\gamma$-tubulin (Sigma-Aldrich, T6557, 1:1000). Following incubations, membranes were washed and incubated in secondary antibodies (LiCor) prior to imaging on a LiCor Odyssey.

\subsection{Statistical analysis.}

Data were analyzed using GraphPad Prism software. Independent and paired T-tests, one-way analysis of variance (ANOVA), or factorial ANOVAs were performed where appropriate. Secondary posthoc analysis found differences in groups using either Tukey or Sidek multiple comparisons were appropriate. $P<0.05$ was considered significant.

\section{RESULTS}

\subsection{Liver-specific knockout of Mogat1}

We generated mice with hepatocyte-specific deletion of Mogat1 by crossing Mogat1 floxed mice [15] to homozygosity with mice expressing albumin promoter-driven Cre recombinase (Figure 1). The resulting offspring mice were outwardly normal on a standard chow diet. When given ad libitum access to a $10 \%$ fat (LFD) or $60 \%$ fat (HFD), the mice gained weight similar to littermate $\mathrm{fl} / \mathrm{fl}$ controls (Figure 1A). Contrary our previous studies showing a beneficial effect of Mogat1 ASO on metabolic parameters in obese mice, glucose and insulin tolerance was not different between genotypes on LFD or 
HFD (Figure 1B, C). We confirmed that Mogat1 expression was markedly suppressed in Mogat1 fl/fl AlbCre+ livers and freshly isolated primary hepatocytes (of note, Mogat1 expression drastically decreases after hepatocyte isolation and plating, data not shown) (Figure 1D, E). Mogat2 tended to be, but was not significantly increased in knockout mice or isolated hepatocytes (Figure 1F, G). Liver weight was not altered by Mogat1 knockout (Figure 1H). Hepatic TAG content increased with HFD but was similar between genotypes (Figure 1I). In contrast to previous work with Mogat1 ASO-treated mice, MGAT activity in isolated liver membranes was not affected by Mogat1 knockout (Figure 1J and ref. [17]).

\subsection{AAV8-mediated liver-specific knockout of Mogat1}

To avoid possible compensation from chronic Mogat1 knockout, we injected Mogat1 fl/fl mice with an AAV8-TBG-Cre or eGFP control after 16 weeks of HFD feeding (Figure 2). After three weeks, AAV8-Cre knockout mice had similar body weight and no differences in glucose or insulin tolerance (Figure 2A-C). The AAV8-Cre reduced Mogat 1 expression but did not affect Mogat2 expression (Figure 2D). Similar to constitutive Mogat1 liver-specific knockout, acute deletion of hepatic Mogat1 did not alter liver weight, TAG content, and actually, slightly increased MGAT activity (Figure 2E-G). Together, these data indicate that genetic deletion of Mogat1 in liver of diet-induced obese mice does not affect glucose or insulin tolerance, and suggests compensation from other enzymes with MGAT activity

\section{$[11,12,25]$.}

\subsection{Mogat1 whole-body deletion}

Mogat1 ASO treatment likely results in gene knockdown in multiple tissues [21]. Thus, we generated Mogat1 whole body null mice (MOKO) (Figure 3). MOKO mice were outwardly normal on standard chow diet. Unexpectedly, when given a HFD, MOKO mice gained more weight than littermate wild-type control mice (Figure 3A, B). The heterozygous littermates had an intermediate phenotype (Figure 3B and data not shown). The increase in weight gain was not attributed to increased adiposity as evident by fat mass and percent adiposity in ECHO MRI and individual fat pad weights and thus may be 
due to larger body size (Figure 3B). Glucose tolerance was not affected by Mogat1 knockout; however, HFD-fed MOKO mice had reduced insulin tolerance and increased plasma insulin concentrations, which could be indicative of insulin resistance (Figure 3D, E). Mogat1 gene expression was undetectable in livers of MOKO mice (Figure 3F) or in any other tissue analyzed (data not shown). Lastly, MOKO mice had larger livers compared to wild-type littermates, but similar TAG content and MGAT activity (Figure $3 G-I)$.

\subsection{Hepatic Mogat1 overexpression}

We next wanted to determine if overexpression of hepatic Mogat1 in the context of LFD or HFD was sufficient to promote hepatic steatosis, insulin resistance, as well as deleterious effects on systemic glucose metabolism. We treated C57BL6/J mice with LFD or HFD for 6 weeks then injected them with AAV8 expressing either eGFP or mouse Mogat1 under control of the hepatocyte-specific TBG promoter (Figure 4). Mogat1 overexpressing (OE) mice gained similar weight as the GFP control-treated mice on either diet (Figure 4A). After 16 weeks of diet (10 weeks of overexpression), there was no difference in glucose or insulin tolerance between groups on HFD (Figure 4B, C). Mogat1 and GFP expression was significantly increased by the administration of each AAV8, respectively and there was no difference in Mogat2 expression between groups (Figure 4D-F). Mogat1 overexpression did not alter liver weight but did increase liver TAG and MGAT activity in the LFD groups (Figure 4H, I). Mogat1 overexpression did not affect MGAT activity in HFD fed mice, likely due to the effect of HFD in increasing MGAT activity. Mogat1 protein expression was restricted to liver membranes where MGAT enzymatic activity occurs (Figure 4J). Thus, we conclude that hepatic Mogat1 overexpression is sufficient to induce hepatic steatosis on a LFD, but not insulin resistance or systemic dysregulation of glucose metabolism.

\section{5 shRNA mediated knockdown of Mogat1}

Work conducted independently of our lab has shown that adenovirus-delivered Mogat1 shRNA to knockdown hepatic Mogat1 expression decreased hepatic triglycerides and plasma glucose in mouse 
models of fatty-liver disease $[26,20]$. As an alternative to our genetic models, we used AAV8 to deliver the same shRNAs targeted against Mogat1 or scramble controls in HFD-fed C57BL6/J mice (Figure 5). However, Mogat1 knockdown by shRNA did not improve glucose or insulin tolerance in HFD fed mice in our study (Figure 5A-C). Mogat1 gene expression was significantly reduced by Mogat1 shRNA treatment, but was not returned to levels of LFD mice, and Mogat1 shRNA did not affect liver weight or TAG levels (Figure 5D-G). These data suggest that shRNA-mediated Mogat1 knockdown does not improve insulin sensitivity or glucose metabolism.

\subsection{Mogat1 ASO treatment improves hepatic insulin sensitivity on HFD}

To confirm our previous reports indicating that Mogat1 suppression by ASO improves hepatic insulin sensitivity $[17,18]$, we fed C57BL6/J mice with a LFD or HFD. After 16 weeks, mice were weightmatched again and randomized to receive ASOs targeting either Mogat1 or scramble control (Figure 6). As previously described [17], Mogat1 ASO treatment lowered blood glucose and plasma insulin concentrations compared to scramble treated controls in HFD fed mice without affecting body weight (Figure 6A-C). Hepatic Mogat1 and Mogat2 gene expression were significantly increased by HFD, but Mogat1 ASO treatment suppressed Mogat1 expression without affecting Mogat2 (Figure 6D). Mogat1 ASO treatment increased liver weight (Figure 6E) without increasing TAG content (Figure 6F). The increased liver weight could be due to glycogen accumulation, which was higher in Mogat1 ASO treated mice (Figure 6G). Together these data confirm our previous reports that Mogat1 ASO treatment increases improves hepatic metabolism.

\subsection{Mogat1 ASO treatment improves glucose tolerance in Mogat1 whole-body null mice}

Given the inability to reproduce the insulin-sensitizing effects of Mogat1 ASOs in any of our genetic or shRNA Mogat1 deletion models, we tested whether Mogat1 ASO treatment improves glucose metabolism independently of Mogat1. We treated MOKO mice, fed a HFD for 16 weeks, with ASOs targeting Mogat1 or scramble controls (Figure 7). Remarkably, Mogat1 ASO treatment improved glucose 
tolerance in both MOKO and WT mice (Figure 7A, B). Furthermore, Mogat1 ASO treatment increased liver weight and liver TAG in WT mice, with a trend in the MOKO groups (Figure 7C, D). Mogat1 ASO treatment suppressed Mogat1 expression in WT mice (Figure 7E).

Recently, McCabe et al. demonstrated that ASO targeting TTC39B non-specifically protects against diet-induced obesity and induces adipose tissue browning through activation of a type I interferon receptor (IFNAR-1) in adipose tissue-derived macrophages, even in TTC39B knockout mice [22]. Although we did not observe any effect on body weight associated with use of the Mogat1 ASO, we found that expression of several key indicators of IFNAR-1 signaling (Oas/1, Ifit1, and IIfit2) were significantly increased in liver of MOKO mice treated with Mogat 1 ASOs (Figure 7F-H). The Mogat1 ASO also tended to increase the expression of these genes in WT mice, while Ifnar1 gene expression was unchanged in all mice (Figure 7l).

\subsection{IFNAR-1 blockade does not prevent improvements in glucose homeostasis in Mogat1 ASO treated} mice.

To determine if the increase in IFNAR-1 activation in response to Mogat1 ASO treatment drives the improvements in glucose metabolism, we injected ASO-treated mice with an IFNAR-1 neutralizing antibody or IgG control during a HFD challenge (Figure 8)[22,27]. We repeated the previous observation that the Mogat1 ASO improved glucose tolerance in HFD fed mice (Figure 8A, B), but co-treatment with IFNAR-1 neutralizing antibody did not block the improvements in glucose tolerance in Mogat1 ASO treated mice (Figure 8A, B). Liver weight and TAG content were unaffected by any treatment (Figure 8C, D). Mogat1 ASO treatment suppressed liver Mogat1 gene expression in both IgG and IFNAR-1

neutralizing antibody treated mice (Figure 8E). Mogat1 ASO again activated IFNAR-1 responsive genes in liver, but each of these effects was blocked by use of the IFNAR-1 neutralizing antibody (Figure 8F-I). Mogat1 ASO treatment also suppressed Mogat1 gene expression (Figure 8J) and increased Ifit/2 gene expression but not Ifit/1, Oas/1, and Ifnar1 in adipose tissue (Figure 8K-N). Lastly, gene expression of the 
WAT beiging marker, Ucp1 was induced by Mogat1 ASO and blocked by IFNAR-1-Ab (Figure 8O). Other markers of WAT beiging were unaffected by either treatment (Ppargc1a or Arb2) (Figure 8P-Q). These data indicate that Mogat1 ASO treatments non-specifically improve glucose metabolism, but this effect is not mediated through the previously implicated mechanism of IFNAR-1 activation [22].

\section{DISCUSSION}

Hepatic steatosis is tightly linked to insulin resistance and development of systemic metabolic abnormalities. Previous work by our group and others using RNA interference approaches has demonstrated that targeting Mogat1 in livers of obese mice improves glucose homeostasis and insulin resistance $[17,18,20,26]$. In the present study, we knocked out Mogat1 specifically in hepatocytes, both chronically and acutely, and also generated a global Mogat1 knockout, but did not observe any improvements in glucose homeostasis or insulin sensitivity in diet-induced obese mice. Hepatic Mogat1 overexpression was sufficient to increase hepatic MGAT activity and TAG content, but only in LFD-fed mice, and did not result in insulin resistance or glucose intolerance. Although we reproduced our previous findings to demonstrate that Mogat1 ASO improves glucose tolerance in obese mice, we also conclusively demonstrate that this ASO still improves metabolic homeostasis in mice with global Mogat1 deletion. Lastly, we confirm these improvements were not due to activation of interferon signaling through IFNAR-1 as recently reported for an ASO against TTC39B [22]. Collectively, these data suggest that Mogat1 ASO treatment improves glucose homeostasis and insulin sensitivity independently of knocking down Mogat1 expression and by mechanisms that remain to be elucidated.

The development of technologies for in vivo RNA interference through use of modified oligonucleotides has been a boon to hepatology and related disciplines. The ease of administration and liver-trophic nature of these designer oligonucleotides has allowed researchers to suppress the expression of a variety of genes in the liver and also led to clinically-approved approaches to treating metabolic disease $[28,29]$. We were quite flummoxed when characterizing the phenotype of mice 
lacking Mogat1 in liver by the inability to phenocopy our previously reported phenotypes obtained by using ASOs $[17,18,30]$ or adenoviral-driven expression of shRNA $[20,26]$. We have considered a number of possibilities to explain this. It is likely that genetic deletion of Mogat1 leads to compensatory changes in other enzymes that have MGAT activity, since ASO treatment leads to reduced MGAT activity in liver [17] whereas MGAT knockout either acutely or chronically does not (Figures 1-3). Another possibility is that the Mogat1 ASO was mediating its effects on a tissue other than liver. Brandon and colleagues recently reported that that liver-specific KO of protein kinase $C \varepsilon(P K C \varepsilon)$ did not phenocopy previous ASO work conducted in rats and found instead that deletion of PKC $\varepsilon$ in adipose tissue, which is often targeted by ASOs as well, produced an insulin sensitive phenotype [31,32]. However, our studies with adipocyte-specific Mogat1 KO mice (manuscript in preparation) or with the global MOKO mice did not reveal an insulin sensitive phenotype and thus, ASOs targeting Mogat1 in other tissues does not explain the observed discrepancies. Taken together with previous work, these studies demonstrate the importance of using rigorous and complementary controls when employing ASOs to study intermediary metabolism and insulin sensitivity.

It is now clear that Mogat1 ASO elicits its effects even in the absence of Mogat1 expression. In this work and in previous studies, we have used multiple ASO sequences targeting Mogat1 with similar effects on glucose metabolism and do not anticipate these effects are due to silencing another gene from off-target interactions with RNA. The present results parallel another recent paper that showed that ASOs targeting TTC39B (T39) still elicited metabolic benefits in global T39 KO mice [22]. McCabe and colleagues [22] demonstrated in their model that ASO treatment activated an interferon signaling pathway as indicated by an induction of IFNAR-1 responsive genes (Oas/1, Ifit1, and Ifit2) that led to adipocyte beiging and caused the mice to lose weight. Mice lacking IFNAR-1 were protected from ASOinduced weight loss and beiging in that study as well. Exactly, how the interferon response is activated by ASO, how this occurs in the KO in the absence of target RNA, and why the scramble control ASO does 
not provoke the same response is still unclear. Although we did not observe weight loss in our studies, Mogat1 ASO treatment in WT or MOKO mice stimulated the expression of Oas/1, Ifit1, and Ifit2 in liver and in adipose tissue also induced the expression of Ucp1 in fat. Moreover, these responses were exaggerated in MOKO mice for reasons yet to be determined. The activation of IFNAR-1 signaling may be consistent with our previous work demonstrating that Mogat1 ASO exacerbates hepatic inflammation on a diet that induces nonalcoholic steatohepatitis, including components of interferon signaling [21]. While IFNAR-1 signaling activates a multitude of signaling pathways that could plausibly affect glucose metabolism and insulin sensitivity [33], we were unable to prevent the improvements in glucose metabolism by blocking IFNAR-1 activation with an antibody. Thus, IFNAR-1 activation is not the mechanism for improved glucose and insulin tolerance in response to Mogat1 ASO, which will require further study.

Based on previous work with the Mogat1 ASO [17,18,30], we were surprised to find that genetic deletion of Mogat1 in liver did not affect hepatic MGAT activity. Several enzymes exhibit MGAT activity, including Mogat2 and Dgat1, and lack of MGAT activity suppression could be due to compensation from these enzymes [10-13]. Mogat2 has a higher specific activity than Mogat1 and in many of the current studies was upregulated by high fat diet feeding. Although we did not find evidence for robust transcriptional activation of these enzymes in the Mogat1 knockout mice, there could be posttranscriptional mechanisms including post-translational modifications that enhance activity. The lack of effect on hepatic MGAT activity is consistent with previous work showing that global Mogat1 KO mice do not have deficits in hepatic MGAT activity [34]. However, our previous work has shown that adipocyte-specific KO mice exhibit reduced adipose tissue MGAT activity, which may be consistent with the very low expression of Mogat2 in adipose tissue [15].

\section{CONCLUSIONS}

Here we provide evidence that Mogat1 ASO treatment improves whole-body metabolism 
through Mogat1-independent effects. Liver-specific Mogat1 ablation does not improve insulin resistance in HFD-fed mice, as observed with Mogat1 ASO treatments $[17,18]$. Moreover, we show novel evidence that whole-body Mogat1 deletion leads to insulin resistance, in the context of diet-induced obesity. Finally, we provide preliminary evidence that Mogat1 ASOs improve glucose intolerance even in global Mogat1 KO mice. These findings also demonstrate that careful consideration should be given for using ASOs to target gene suppression, including use of genetic knockout models, in metabolic studies that could be affected by these off-target effects.

ACKNOWLEDGEMENTS: The authors would like to thank Dr. Kyle McCommis at St. Louis University for his insight into off-target effects of ASO treatments, Dr. Eric Yen at the University of Wisconsin for his advice on MGAT biology, and Daniel Ferguson for editing the manuscript during quarantine. We also thank the Washington University School of Medicine Nutrition and Obesity Research Center for continued support in research. Work in the authors' lab was supported by grants from the NIH (R56 DK111735) and the American Diabetes Association (1-17-IBS-109) to BNF and core laboratories of Washington University School of Medicine (Diabetes Research Center (P30 DK020579), Digestive Diseases Research Cores Center, (P30 DK052574), and the Nutrition Obesity Research Center (P30 DK056341). 


\section{REFERENCES}

[1] Adams, L.A., Lymp, J.F., St Sauver, J., Sanderson, S.O., Lindor, K.D., Feldstein, A., et al., 2005. The natural history of nonalcoholic fatty liver disease: a population-based cohort study. Gastroenterology 129(1): 113-21, Doi: 10.1053/j.gastro.2005.04.014.

[2] Fabbrini, E., Sullivan, S., Klein, S., 2010. Obesity and nonalcoholic fatty liver disease: Biochemical, metabolic, and clinical implications. Hepatology 51(2): 679-89, Doi: 10.1002/hep.23280.

[3] Fabbrini, E., Yoshino, J., Yoshino, M., Magkos, F., Luecking, C.T., Samovski, D., et al., 2015. Metabolically normal obese people are protected from adverse effects following weight gain. The Journal of Clinical Investigation 125(2): 787-95, Doi: 10.1172/JCl78425.

[4] Lambert, J.E., Ramos-Roman, M.A., Browning, J.D., Parks, E.J., 2014. Increased De Novo Lipogenesis Is a Distinct Characteristic of Individuals With Nonalcoholic Fatty Liver Disease. Gastroenterology 146(3): 726-35, Doi: 10.1053/j.gastro.2013.11.049.

[5] Smith, G.I., Shankaran, M., Yoshino, M., Schweitzer, G.G., Chondronikola, M., Beals, J.W., et al., 2020. Insulin resistance drives hepatic de novo lipogenesis in nonalcoholic fatty liver disease. The Journal of Clinical Investigation 130(3): 1453-60, Doi: 10.1172/JCI134165.

[6] Buzzetti, E., Pinzani, M., Tsochatzis, E.A., 2016. The multiple-hit pathogenesis of non-alcoholic fatty liver disease (NAFLD). Metabolism 65(8): 1038-48, Doi: 10.1016/j.metabol.2015.12.012.

[7] Coleman, R.A., Lee, D.P., 2004. Enzymes of triacylglycerol synthesis and their regulation. Progress in Lipid Research 43(2): 134-76.

[8] Chon, S.-H., Zhou, Y.X., Dixon, J.L., Storch, J., 2007. Intestinal monoacylglycerol metabolism: developmental and nutritional regulation of monoacylglycerol lipase and monoacylglycerol acyltransferase. The Journal of Biological Chemistry 282(46): 33346-57, Doi: 10.1074/jbc.M706994200.

[9] Han, G.-S., Wu, W.-I., Carman, G.M., 2006. The Saccharomyces cerevisiae Lipin homolog is a Mg2+dependent phosphatidate phosphatase enzyme. The Journal of Biological Chemistry 281(14): 9210 8, Doi: 10.1074/jbc.M600425200.

[10] Cao, J., Burn, P., Shi, Y., 2003. Properties of the mouse intestinal acyl-CoA:monoacylglycerol acyltransferase, MGAT2. The Journal of Biological Chemistry 278(28): 25657-63, Doi: 10.1074/jbc.M302835200.

[11] Yen, C.-L.E., Stone, S.J., Cases, S., Zhou, P., Farese, R.V., 2002. Identification of a gene encoding MGAT1, a monoacylglycerol acyltransferase. Proceedings of the National Academy of Sciences of the United States of America 99(13): 8512-7, Doi: 10.1073/pnas.132274899.

[12] Yen, C.-L.E., Farese, R.V., 2003. MGAT2, a Monoacylglycerol Acyltransferase Expressed in the Small Intestine. Journal of Biological Chemistry 278(20): 18532-7, Doi: 10.1074/jbc.M301633200.

[13] Cases, S., Smith, S.J., Zheng, Y.W., Myers, H.M., Lear, S.R., Sande, E., et al., 1998. Identification of a gene encoding an acyl CoA:diacylglycerol acyltransferase, a key enzyme in triacylglycerol synthesis. Proceedings of the National Academy of Sciences of the United States of America 95(22): 13018-23, Doi: 10.1073/pnas.95.22.13018.

[14] Ho, S.-Y., Storch, J., 2001. Common mechanisms of monoacylglycerol and fatty acid uptake by human intestinal Caco-2 cells. American Journal of Physiology-Cell Physiology 281(4): C1106-17, Doi: 10.1152/ajpcell.2001.281.4.C1106.

[15] Liss, K.H.H., Lutkewitte, A.J., Pietka, T., Finck, B.N., Franczyk, M., Yoshino, J., et al., 2018. Metabolic importance of adipose tissue monoacylglycerol acyltransferase 1 in mice and humans. Journal of Lipid Research: jlr.M084947, Doi: 10.1194/jlr.M084947.

[16] Hall, A.M., Kou, K., Chen, Z., Pietka, T.A., Kumar, M., Korenblat, K.M., et al., 2012. Evidence for regulated monoacylglycerol acyltransferase expression and activity in human liver. Journal of Lipid Research 53(5): 990-9, Doi: 10.1194/jlr.P025536. 
[17] Hall, A.M., Soufi, N., Chambers, K.T., Chen, Z., Schweitzer, G.G., McCommis, K.S., et al., 2014. Abrogating Monoacylglycerol Acyltransferase Activity in Liver Improves Glucose Tolerance and Hepatic Insulin Signaling in Obese Mice. Diabetes 63(7): 2284-96, Doi: 10.2337/db13-1502.

[18] Soufi, N., Hall, A.M., Chen, Z., Yoshino, J., Collier, S.L., Mathews, J.C., et al., 2014. Inhibiting Monoacylglycerol Acyltransferase 1 Ameliorates Hepatic Metabolic Abnormalities but Not Inflammation and Injury in Mice. Journal of Biological Chemistry 289(43): 30177-88, Doi: 10.1074/jbc.M114.595850.

[19] Hayashi, Y., Suemitsu, E., Kajimoto, K., Sato, Y., Akhter, A., Sakurai, Y., et al., 2014. Hepatic Monoacylglycerol O-acyltransferase 1 as a Promising Therapeutic Target for Steatosis, Obesity, and Type 2 Diabetes. Molecular Therapy - Nucleic Acids 3, Doi: 10.1038/mtna.2014.4.

[20] Yu, J.H., Song, S.J., Kim, A., Choi, Y., Seok, J.W., Kim, H.J., et al., 2016. Suppression of PPARYmediated monoacylglycerol $O$-acyltransferase 1 expression ameliorates alcoholic hepatic steatosis. Scientific Reports 6: 29352, Doi: 10.1038/srep29352.

[21] Geary, R., Norris, D., Bennett, F., Rosie, Y., 2015. Pharmacokinetics, biodistribution and cell uptake of antisense oligonucleotides. Advanced Drug Delivery Reviews 87: 46-51, Doi: 10.1016/j.addr.2015.01.008.

[22] McCabe, K.M., Hsieh, J., Thomas, D.G., Molusky, M.M., Tascau, L., Feranil, J.B., et al., 2020. Antisense oligonucleotide treatment produces a type I interferon response that protects against diet-induced obesity. Molecular Metabolism, Doi: 10.1016/j.molmet.2020.01.010.

[23] Wilson, E.B., Yamada, D.H., Elsaesser, H., Herskovitz, J., Deng, J., Cheng, G., et al., 2013. Blockade of Chronic Type I Interferon Signaling to Control Persistent LCMV Infection. Science 340(6129): 202-7, Doi: $10.1126 /$ science.1235208.

[24] McCommis, K.S., Chen, Z., Fu, X., McDonald, W.G., Colca, J.R., Kletzien, R.F., et al., 2015. Loss of Mitochondrial Pyruvate Carrier 2 in the Liver Leads to Defects in Gluconeogenesis and Compensation via Pyruvate-Alanine Cycling. Cell Metabolism 22(4): 682-94, Doi: 10.1016/j.cmet.2015.07.028.

[25] Cao, J., Cheng, L., Shi, Y., 2007. Catalytic properties of MGAT3, a putative triacylgycerol synthase. Journal of Lipid Research 48(3): 583-91, Doi: 10.1194/jIr.M600331-JLR200.

[26] Lee, Y.J., Ko, E.H., Kim, J.E., Kim, E., Lee, H., Choi, H., et al., 2012. Nuclear receptor PPARy-regulated monoacylglycerol O-acyltransferase 1 (MGAT1) expression is responsible for the lipid accumulation in diet-induced hepatic steatosis. Proceedings of the National Academy of Sciences of the United States of America 109(34): 13656-61, Doi: 10.1073/pnas.1203218109.

[27] Wilson, E.B., Yamada, D.H., Elsaesser, H., Herskovitz, J., Deng, J., Cheng, G., et al., 2013. Blockade of Chronic Type I Interferon Signaling to Control Persistent LCMV Infection. Science 340(6129): 202-7, Doi: $10.1126 /$ science.1235208.

[28] Khvorova, A., Watts, J.K., 2017. The chemical evolution of oligonucleotide therapies of clinical utility. Nature Biotechnology 35(3): 238-48, Doi: 10.1038/nbt.3765.

[29] Rossor, A.M., Reilly, M.M., Sleigh, J.N., 2018. Antisense oligonucleotides and other genetic therapies made simple. Practical Neurology 18(2): 126-31, Doi: 10.1136/practneurol-2017-001764.

[30] Lutkewitte, A.J., McCommis, K.S., Schweitzer, G.G., Chambers, K.T., Graham, M.J., Wang, L., et al., 2019. Hepatic Monoacylglycerol Acyltransferase 1 is Induced by Prolonged Food Deprivation to Modulate the Hepatic Fasting Response. Journal of Lipid Research: jlr.M089722, Doi: 10.1194/jlr.M089722.

[31] Samuel, V.T., Liu, Z.-X., Wang, A., Beddow, S.A., Geisler, J.G., Kahn, M., et al., 2007. Inhibition of protein kinase $C \varepsilon$ prevents hepatic insulin resistance in nonalcoholic fatty liver disease. Journal of Clinical Investigation 117(3): 739-45, Doi: 10.1172/JCI30400. 
[32] Brandon, A.E., Liao, B.M., Diakanastasis, B., Parker, B.L., Raddatz, K., McManus, S.A., et al., 2019. Protein Kinase C Epsilon Deletion in Adipose Tissue, but Not in Liver, Improves Glucose Tolerance. Cell Metabolism 29(1): 183-191.e7, Doi: 10.1016/j.cmet.2018.09.013.

[33] Dodington, D.W., Desai, H.R., Woo, M., 2018. JAK/STAT - Emerging Players in Metabolism. Trends in Endocrinology \& Metabolism 29(1): 55-65, Doi: 10.1016/j.tem.2017.11.001.

[34] Agarwal, A.K., Tunison, K., Dalal, J.S., Yen, C.-L.E., Farese, R.V., Horton, J.D., et al., 2016. Mogat1 deletion does not ameliorate hepatic steatosis in lipodystrophic (Agpat2-/-) or obese (ob/ob) mice. Journal of Lipid Research 57(4): 616-30, Doi: 10.1194/jlr.M065896. 


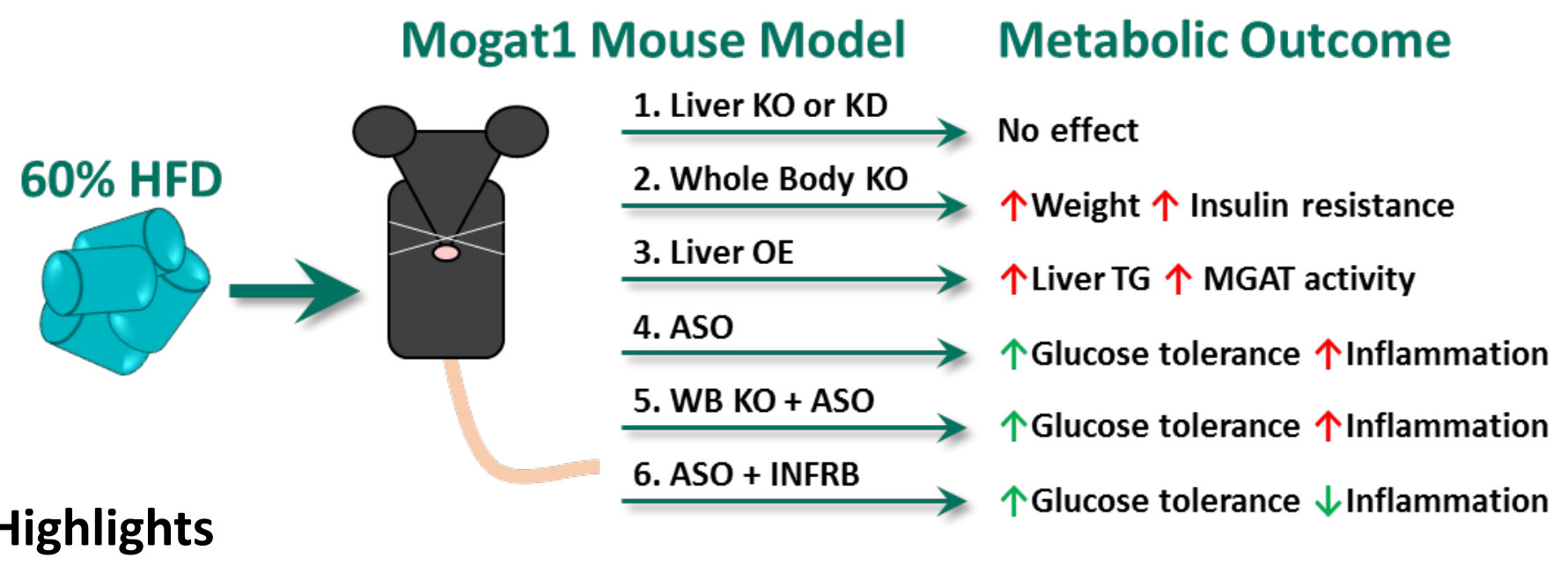

- Mogat1 liver-specific KO or KD does not improve metabolism in HFD fed mice.

- Whole-body Mogat1-deletion impairs insulin tolerance in HFD fed mice.

- Mogat1 ASOs improves whole body metabolism independently of gene knockdown.

- Blockade of the INFR response does not prevent off-target effects of Mogat1 ASOs. 

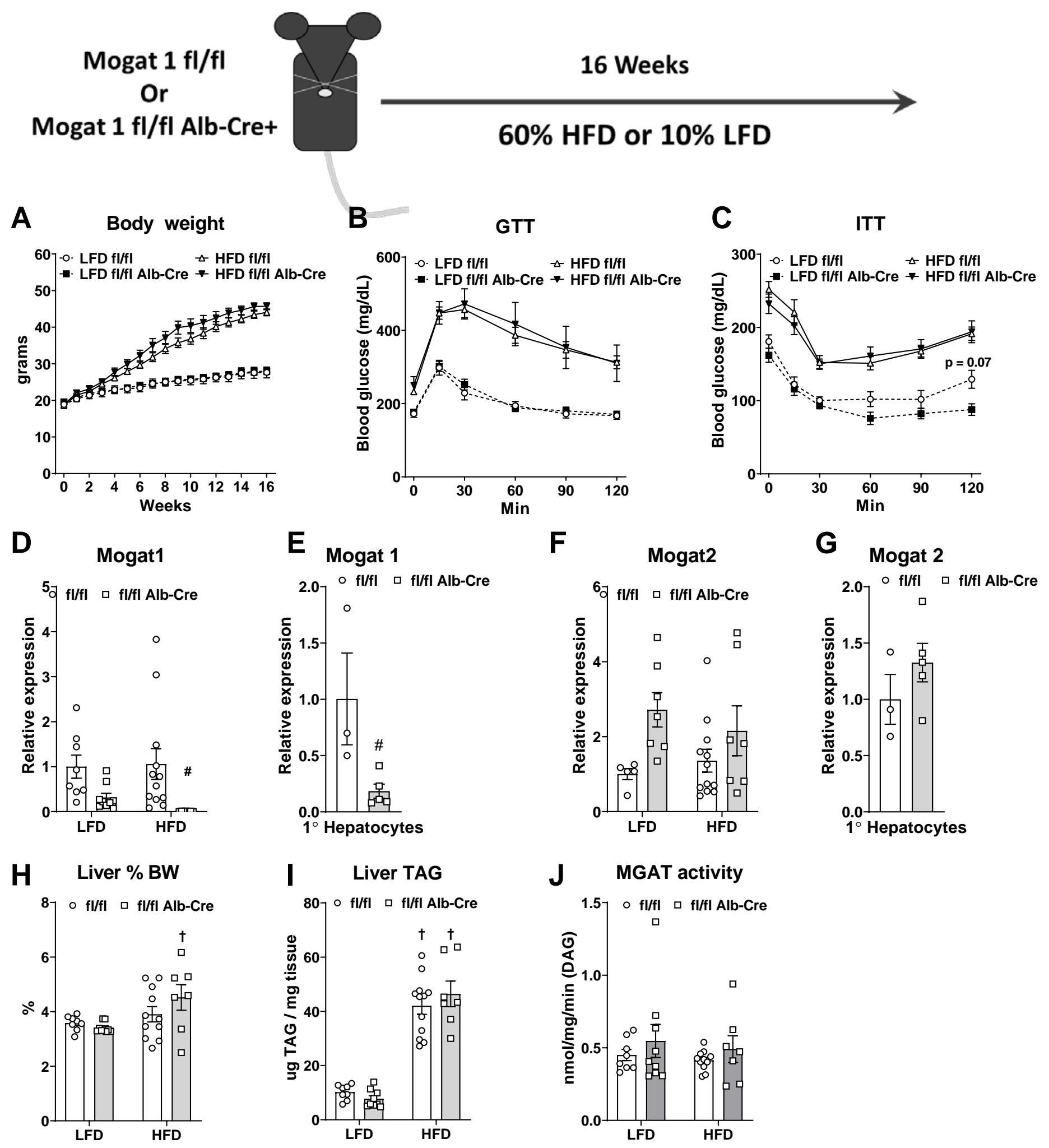

Figure 1. Constitutive liver-specific Mogat1 deletion does not improve insulin sensitivity in mice. Male Mogat1 $\mathrm{fl} / \mathrm{fl}$ mice and littermate Mogat1 $\mathrm{fl} / \mathrm{fl}$ albumin Cre+ mice were fed a LFD or a HFD starting at eight weeks of age for 16 weeks. Mice were fasted for 4 hours prior to sacrifice and tissue collection. A: HFD increased bodyweight in both groups. B,C: HFD fed mice have impaired glucose and insulin tolerance compare to LFD groups. D-G: Mogat1 gene expression is reduced in knockout liver and primary hepatocytes without significant compensation of Mogat2. H,I: HFD increased liver weight and TAG content in both genotypes. J: MGAT activity was not affected by diet or genotype. Data are expressed as means \pm S.E.M. \# $p<0.05$ gene effect, $+p<0.05$ diet effect; $n=5$-10 for mouse studies, $n=3-5$ female mice for primary hepatocyte isolations. 

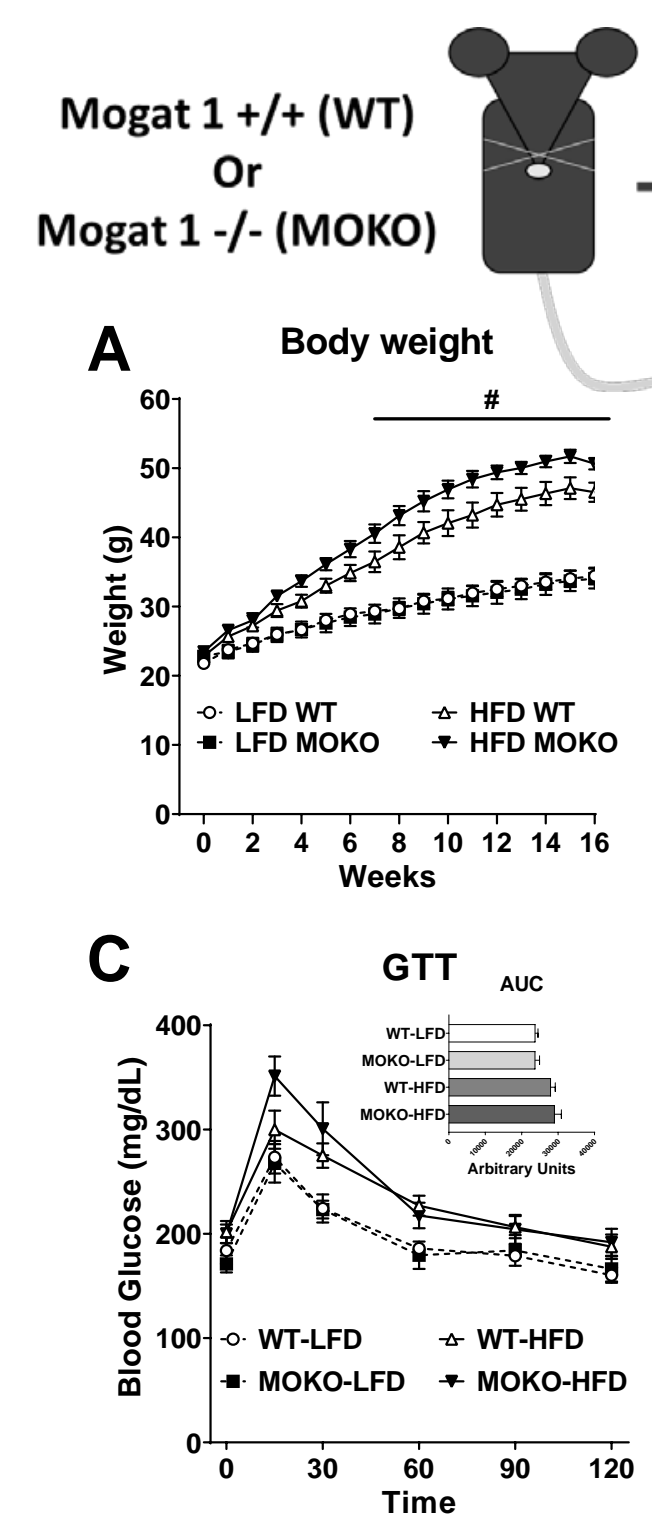

B

\section{$60 \%$ HFD or $10 \%$ LFD}

\section{Weeks}
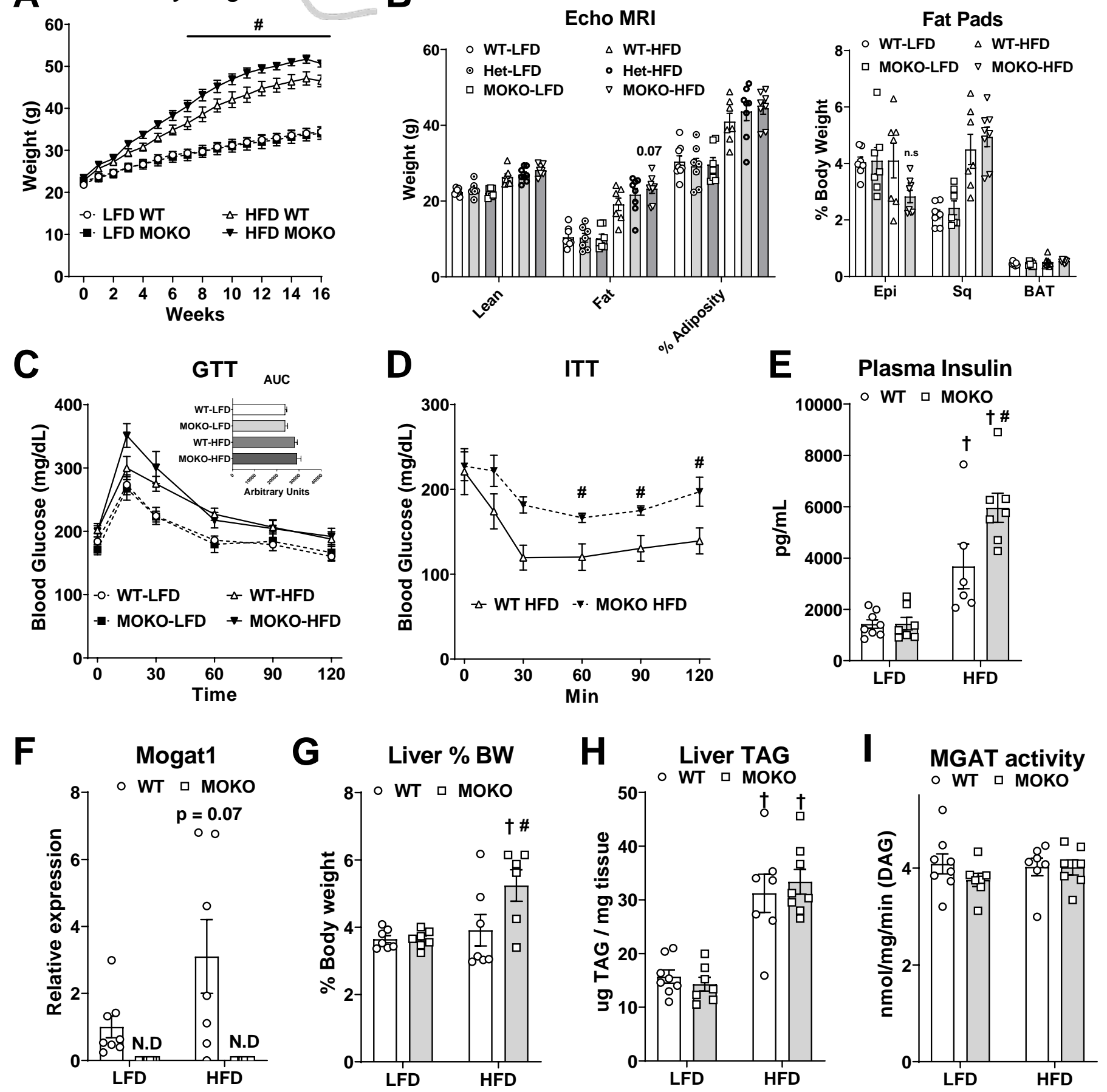

Figure 3. Whole-body deletion of Mogat1 causes weight gain and insulin intolerance on a HFD. Male wild-type (WT) and littermate Mogat1 whole body knockout (MOKO) mice were fed a LFD or a HFD starting at eight weeks of age for 16 weeks. Mice were fasted for 4 hours prior to sacrifice and tissue collection. A: Mogat1 knockout mice gain more weight on a HFD than littermate WT controls. B: ECHO MRI indicates MOKO mice have increased whole body mass, while the heterozygous mice had an intermediate phenotype compared to WT controls. C: Glucose tolerance (dosed on lean mass) was not significantly change in MOKO mice. D,E: HFD fed MOKO mice had significantly impaired insulin tolerance and plasma insulin levels compared to WT controls. F: Mogat1 gene expression was not detectable in MOKO mice. G,H: Liver weight but not TAG was increased in MOKO mice fed a HFD compared to LFD controls. I: MGAT activity was unaffected by either diet or genotype. Data are expressed as means \pm S.E.M. \# $p<0.05$ gene effect, $\dagger p<0.05$ diet effect; $n=6-7$. 
bioRxiv preprint doi: https://doi.org/10.1101/2020.08.05.238535; this version posted August 6, 2020. The copyright holder for this preprint (which was not certified by peer review) is the author/funder. All rights reserved. No reuse allowed without permission.
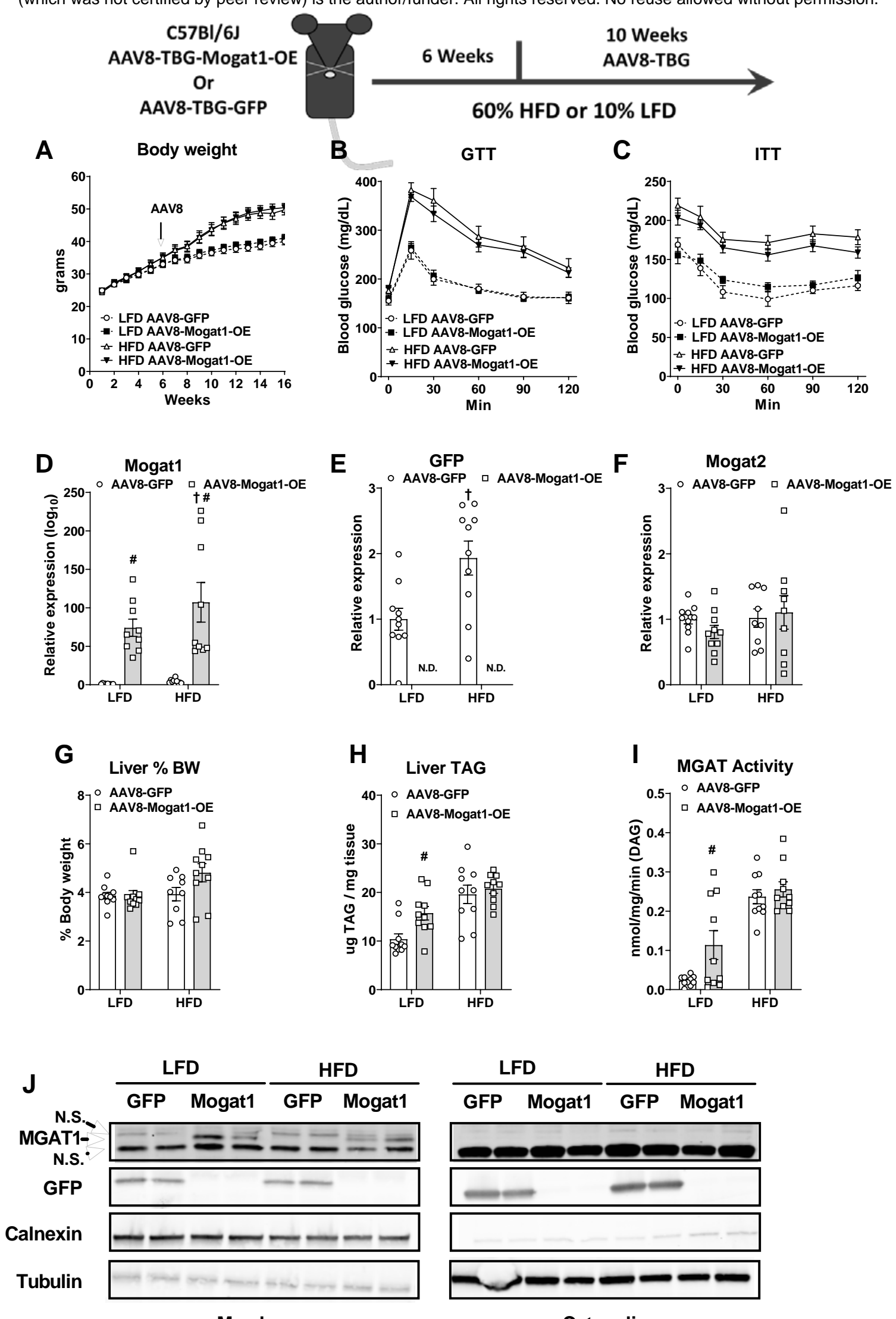

Membrane

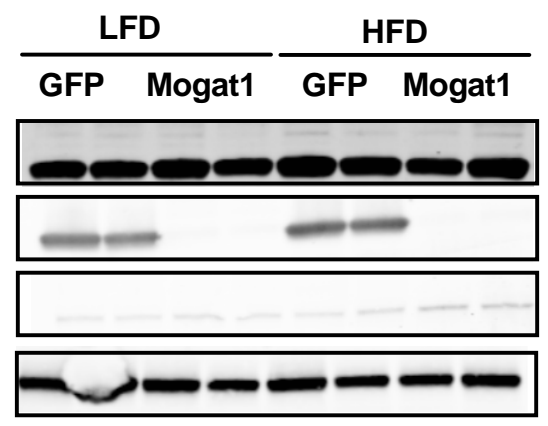

Cytosolic

Figure 4. Hepatic Mogat1 overexpression increases liver TAG and MGAT activity in mice fed a LFD. Male C57BL6/J mice were fed LFD or a HFD starting at eight weeks of age. After 6 weeks of diet mice were injected (retro-orbitally) with AAV8-TGB-eGFP- or AAV8TGB-Mogat1 ( $2 \times 10^{11} \mathrm{GC}$ per mouse) and remained on diet for an additional 10 weeks. Mice were fasted for 4 hours prior to sacrifice and tissue collection. A: HFD fed mice gained weight compare to LFD fed mice in both treatment groups. B,C: Mogat1 overexpression did not impair glucose or insulin tolerance. D-F: Mogat1 and GFP gene expression was significantly increased in AAV8-Mogat1 and AAV8-GFP treated mice, respectively without a change in Mogat2 gene expression. G: Liver weight was unaffected by any treatment. H,I: AAV8-Mogat1 overexpression increased both liver TAG and MGAT activity in LFD mice. J: Western blot analysis indicated Mogat1 and eGFP protein increased in membrane fractions of AAV8 treated livers. Data are expressed as means \pm S.E.M. $\# p<0.05$ gene effect, $+p<0.05$ diet effect; $n=8-10$. 


\section{C57BI/6J \\ Mogat 1 AAV8-ShRNA Or \\ Scramble AAV8-ShRNA}

\section{Weeks}

\section{$60 \%$ HFD or $10 \%$ LFD}
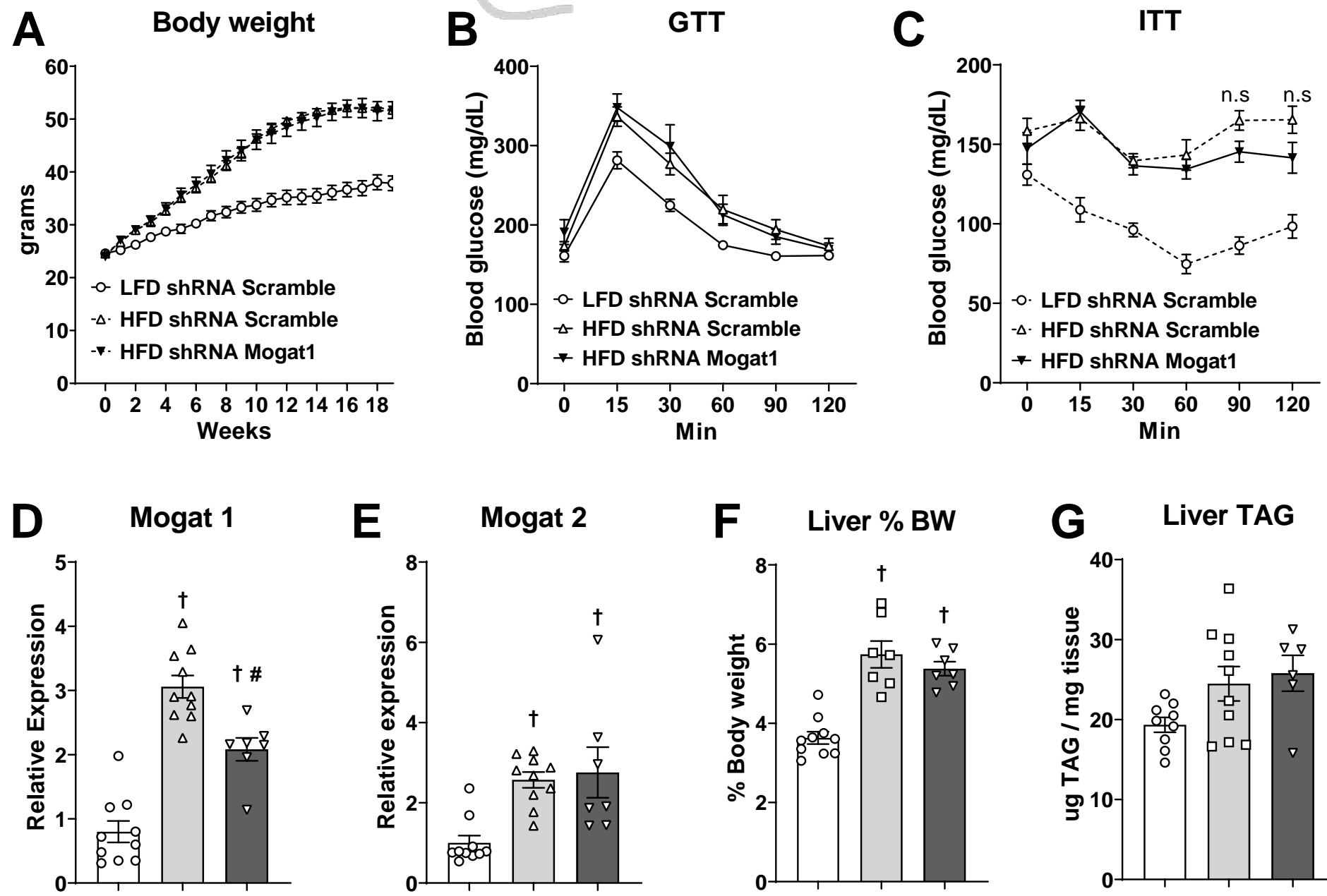

Mogat 2

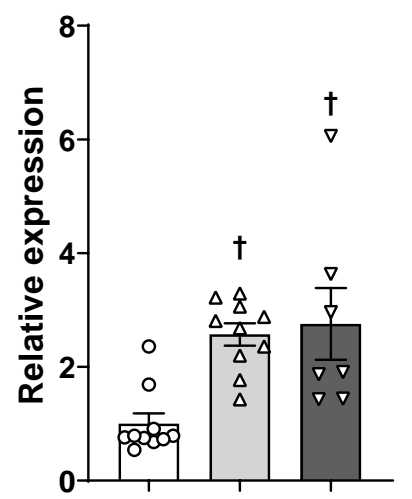

F Liver \% BW

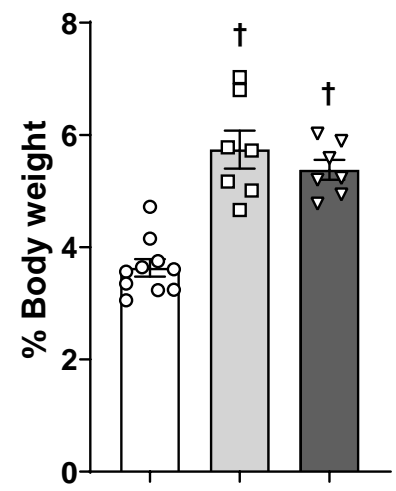

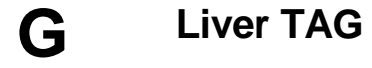

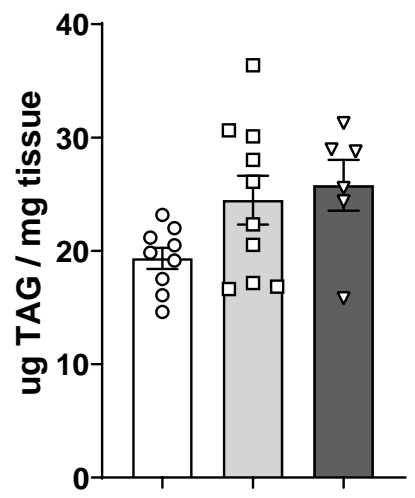

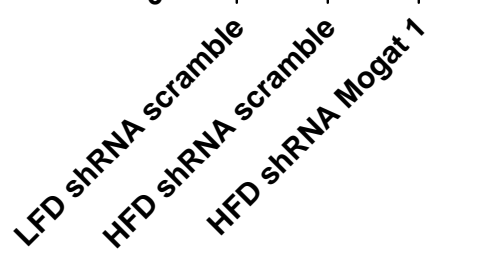
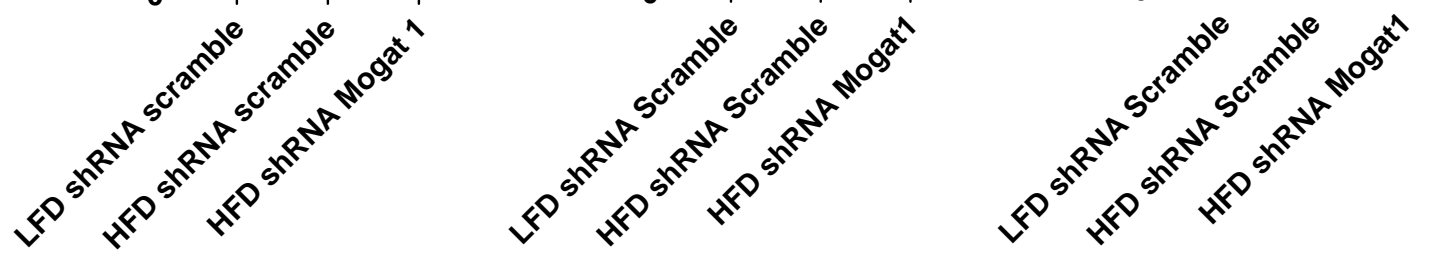

Figure 5. shRNA mediated Mogat1 knockdown did not improve glucose or insulin tolerance on a HFD. Male C57BI/6J mice were fed a $10 \%$ LFD or a $60 \%$ HFD starting at eight weeks of age. After 16 weeks of diet mice were injected (retro-orbitally) with AAV8-U6-shRNA Scramble or shRNAs targeted against Mogat1 $\left(2 \times 10^{11} \mathrm{GC}\right.$ per mouse) and remained on diet for an additional three weeks. Mice were fasted for 4 hours prior to sacrifice and tissue collection. A: HFD fed mice gained weight compare to LFD Scramble treated controls. $B, C$ : HFD fed mice had impaired glucose and insulin tolerance compared to LFD Scramble control treated mice. D,E: Liver weight and TAG were increased by the HFD but unaffected by Mogat1 shRNA treatment. F,G: Mogat1 ShRNA treatment reduced Mogat1 expression but did not affect Mogat2 expression. Data are expressed as means \pm S.E.M. $\# p<0.05$ from HFD shRNA Scramble control mice, $+P<0.05$ from LFD shRNA Scramble controls; $n=7-10$. 
bioRxiv preprint doi: https://doj.org/10.1101/2020.08.05.238535; this version posted August 6, 2020. The copyright holder for this preprint

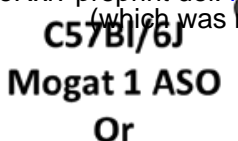

Scramble ASO
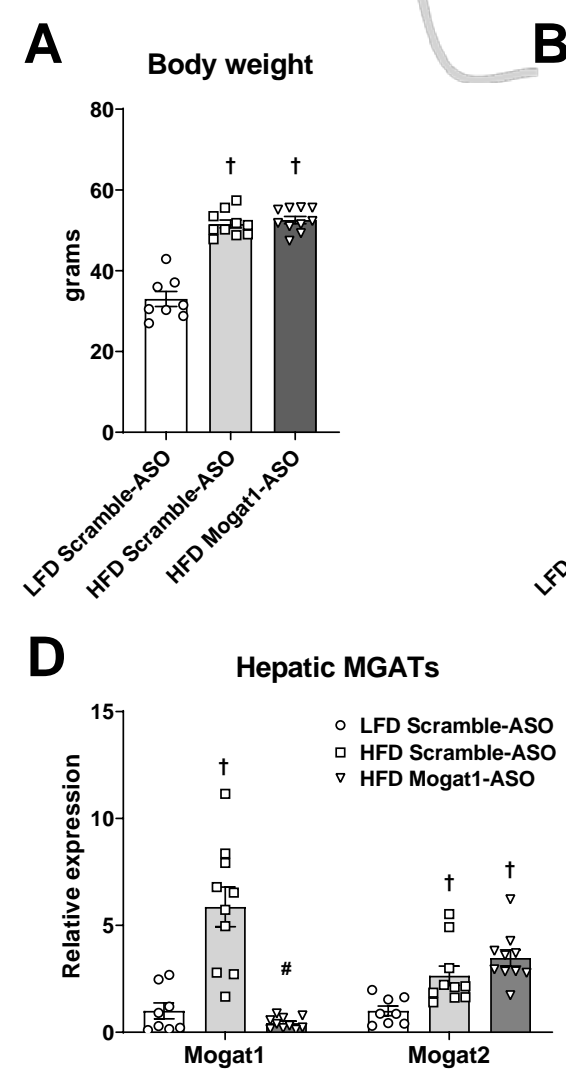

B
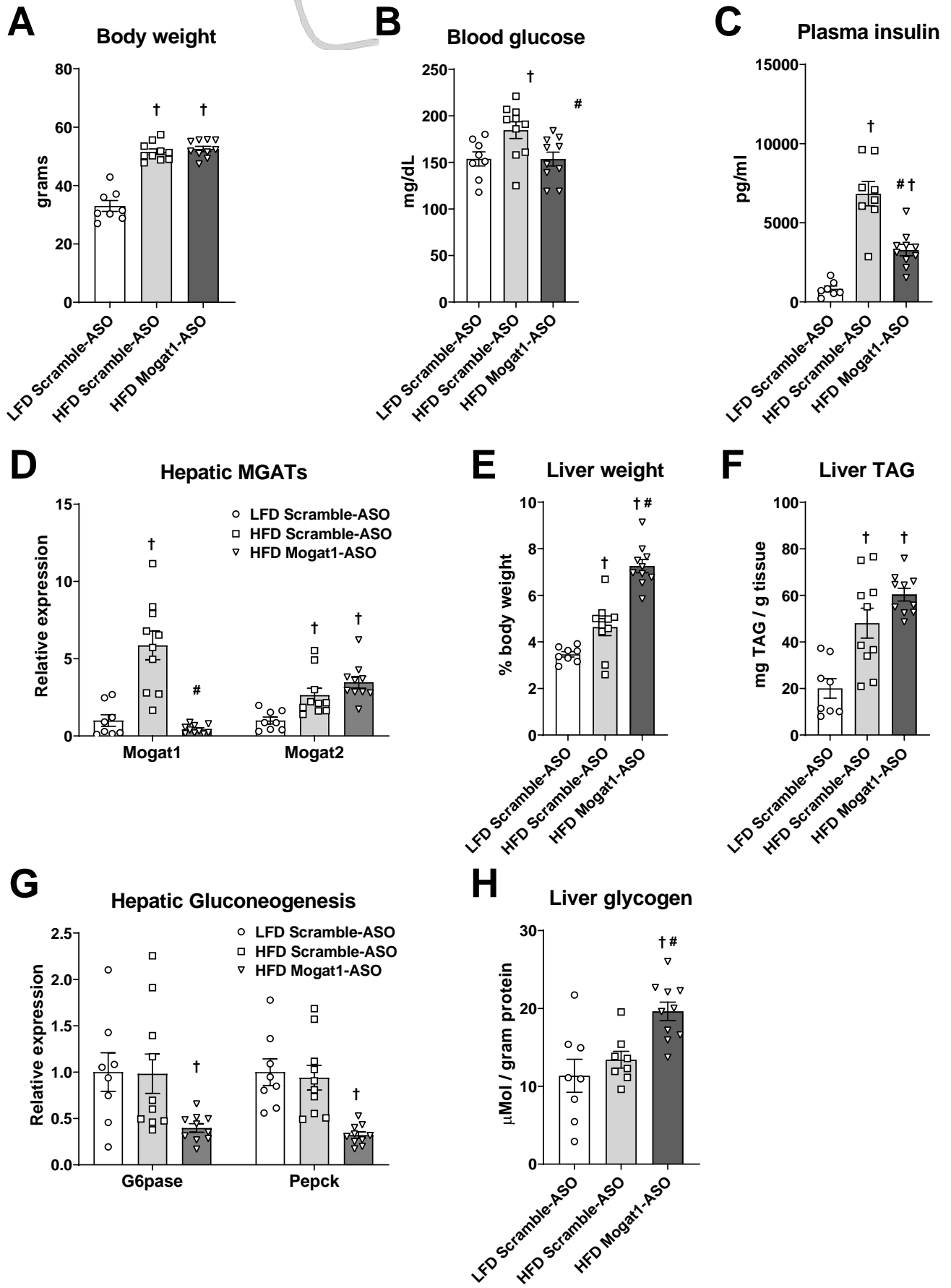

Figure 6. Mogat1 antisense oligonucleotide (ASO, sequence 1) treatment improves insulin sensitivity in HFD fed mice. Male C57BI/6J mice were fed a LFD or a HFD starting at eight weeks of age. After 16 weeks of diet, mice were injected (intraperitoneally) twice weekly with ASOs targeted against Mogat1 or scramble control $(25 \mathrm{mg} / \mathrm{Kg}$ ) for three weeks. Mice were fasted for 4 hours prior to sacrifice and tissue collection. A: HFD fed mice gained more weight compare to LFD fed controls. B,C: Control ASO treated mice had significantly higher blood glucose and plasma insulin than LFD Control ASO treated mice and HFD Mogat1 ASO treated mice. D: Mogat1 ASO treatment significantly reduced hepatic expression of Mogat1, but not Mogat2 as measured by RTqPCR. E: Mogat1 ASO treatment increased liver weight (\%BW) on HFD. F: Liver triglycerides (TAG) were measured enzymatically and were increased by the HFD. G: Hepatic gene expression of Gpase, Pepck, and Pygl were reduced in the HFD Mogat1 ASO treated mice. H: Glycogen was extracted and measure enzymatically and increased in HFD Mogat1 ASO treated mice. Data are expressed as means \pm S.E.M. $\# p<0.05$ ASO effect, $+p<0.05$ diet effect; $n=7-10$. 

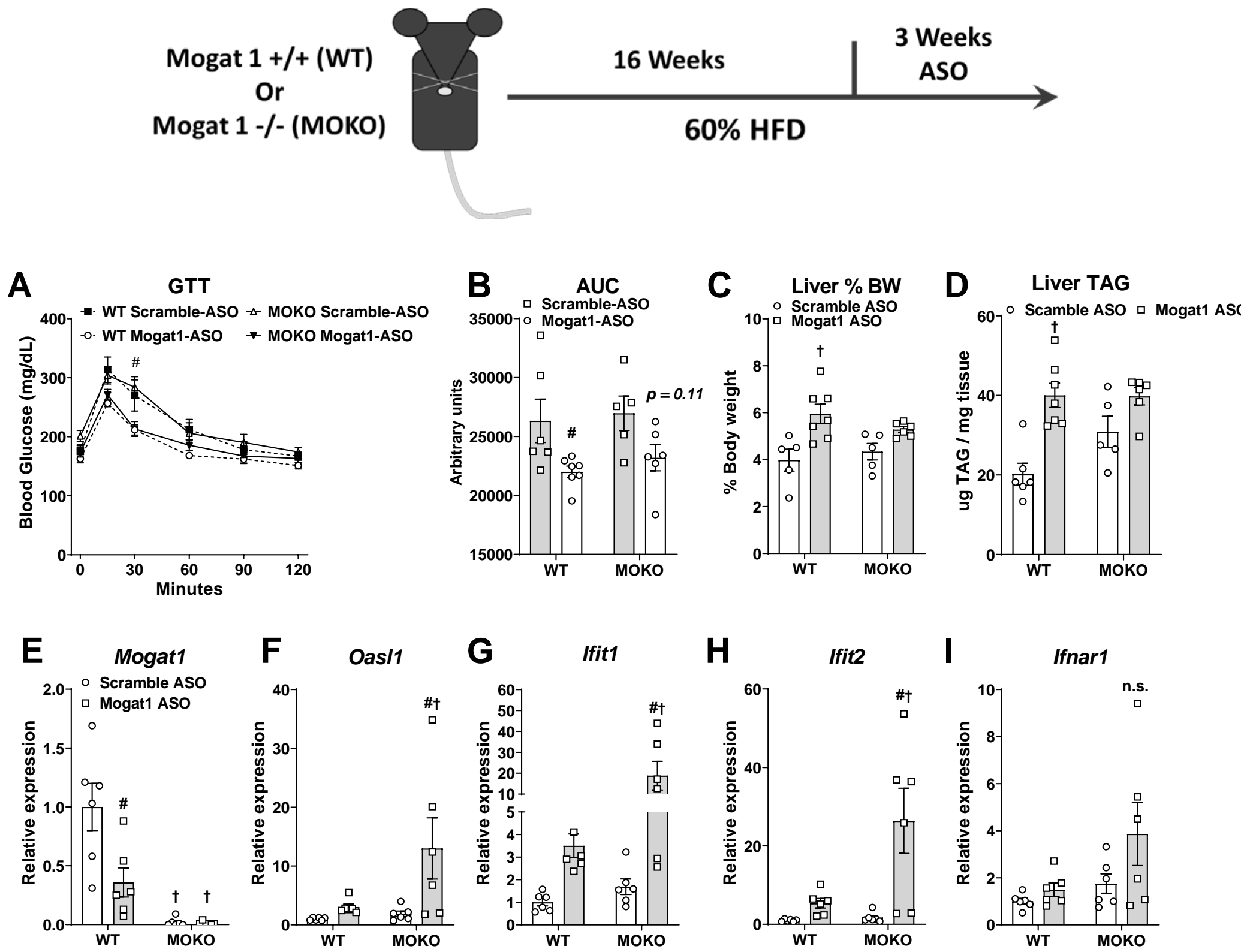

Figure 7. Mogat1 ASO treatment improves glucose tolerance in whole-body Mogat1 null mice on a HFD. Male wild-type (WT) and littermate Mogat1 whole body knockout (MOKO) mice were fed a HFD starting at eight weeks of age. After 16 weeks of diet, mice were injected (intraperitoneally) twice weekly with ASOs targeted against Mogat1 or scramble control ( $25 \mathrm{mg} / \mathrm{Kg})$ for three weeks. Mice were fasted for 4 hours prior to sacrifice and tissue collection. A,B: Mogat1 ASO treatment improves glucose tolerance despite Mogat1 expression. C,D: Mogat1 ASO treatment causes hepatomegaly and increases liver TAG in wild-type mice on HFD. E: Mogat1 gene expression was reduced by ASO treatment and nearly absent in MOKO livers. F-I: Gene expression markers of interferon signaling are increased in Mogat1 ASO treated MOKO mice on HFD. Data are expressed as means \pm S.E.M. \# $p<0.05$ from Scramble ASO $+p<0.05$ from WT controls; $n=5-7$. 
bioRxiv preprint doi: https://doi.org/10.1101/2020.08.05.238535; this version posted August 6, 2020. The copyright holder for this preprint C57BI/6) (which was otertilied by peer review) is the author/funder. All rights reserved. No reuse allowed without permission.

Mogat1 ASO

Or

Scramble ASO

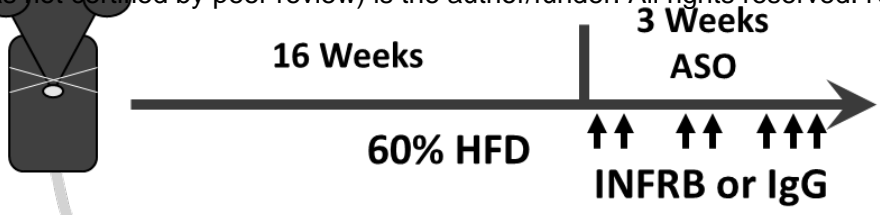

A

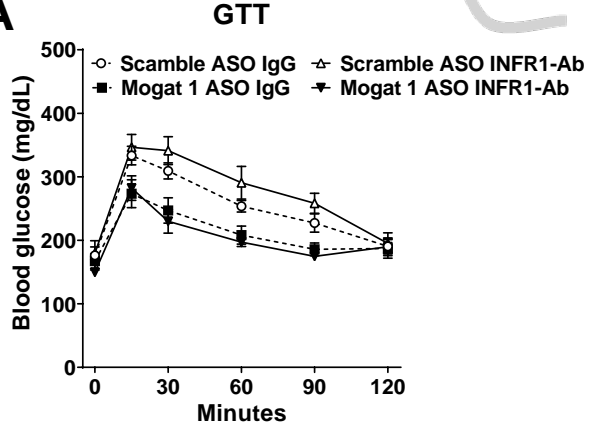

E

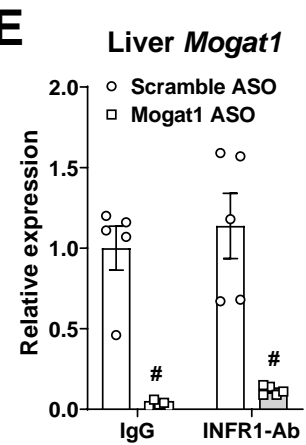

J

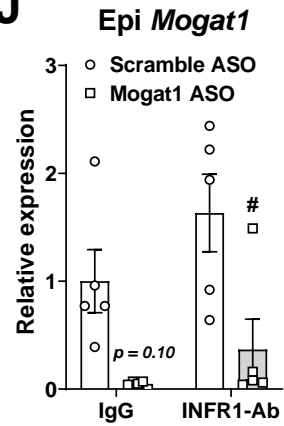

0
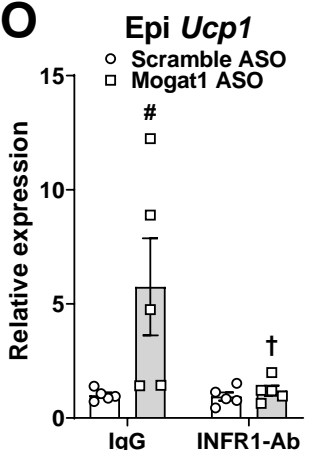

F Liver Oasl1
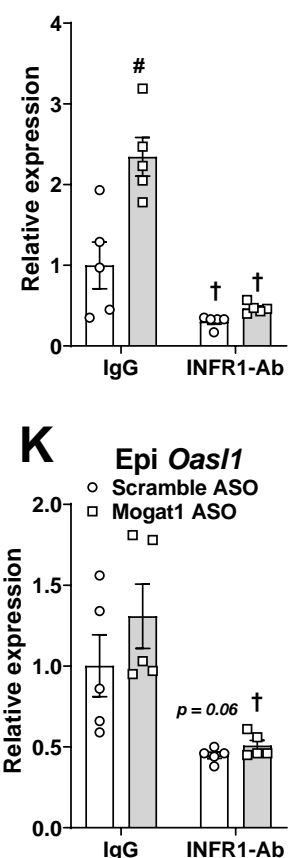

P Epi Ppargc1a

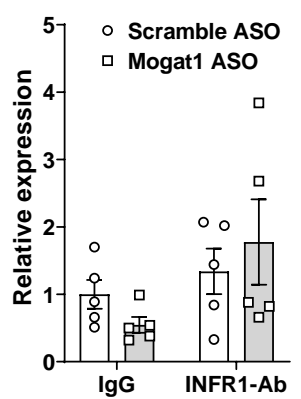

B

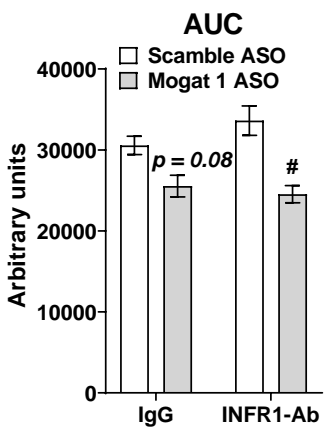

G
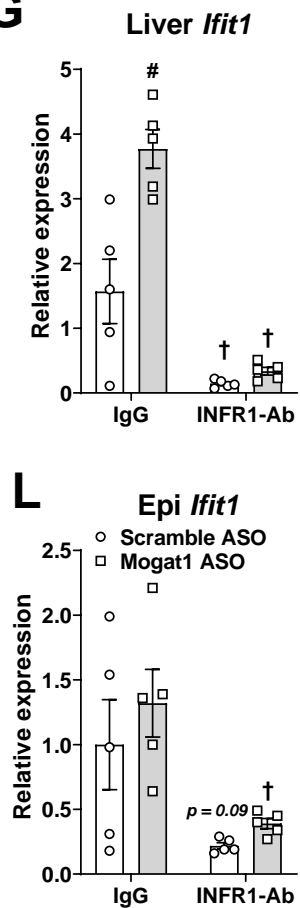

Q

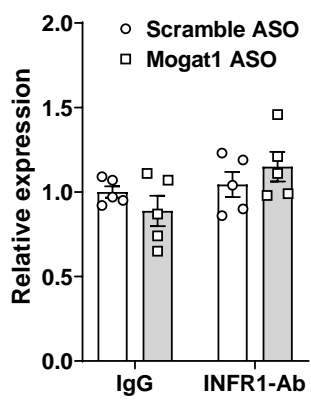

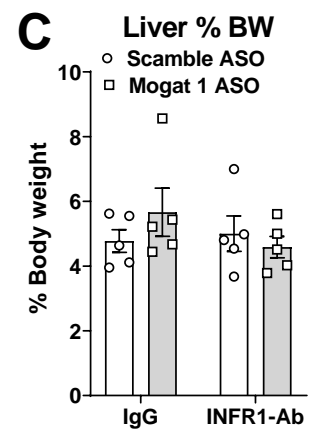

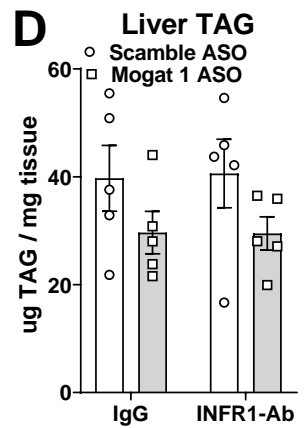

H
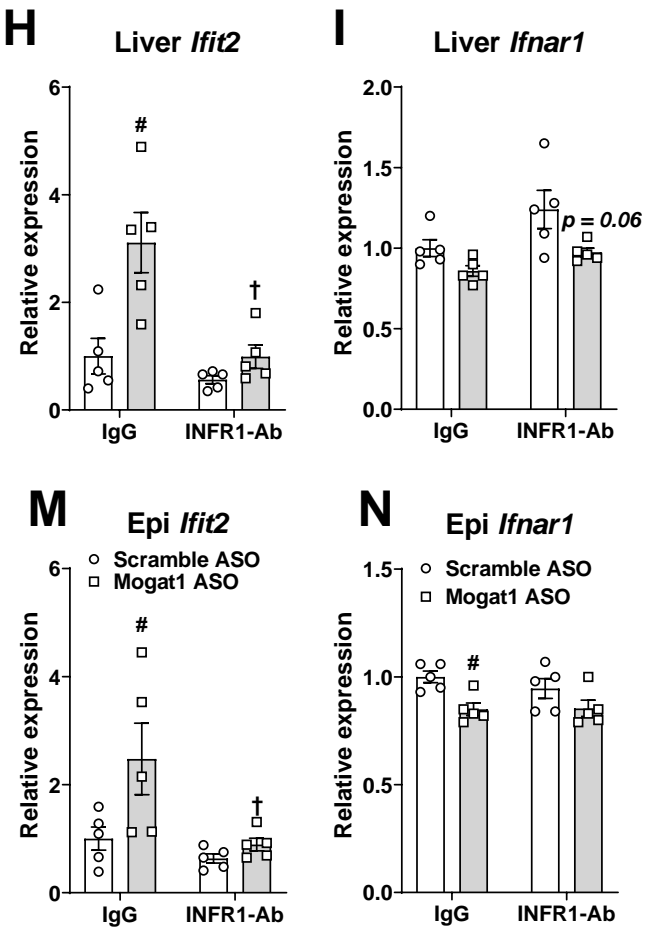

Figure 8. Type I interferon receptor (INFR-1) neutralizing antibody prevents inflammation without effecting glucose tolerance in mice fed a HFD. Male C57BL/6J mice were fed a HFD starting at eight weeks of age. After 16 weeks of diet, mice were injected (intraperitoneally) twice weekly with ASOs targeted against Mogat1 (sequence 2) or scramble control (25 mg/Kg) with an INFR-1 neutralizing antibody (INFR-Ab) or IgG control (250 ug per mouse twice a week for weeks 1\&2, and 500 ug per mouse 3 times for week 3). Mice were fasted for 4 hours prior to sacrifice and tissue collection. A,B: Mogat1 ASO treatment improves glucose tolerance despite INFR-1 antibody treatment. C,D: Liver weight and TAG were unaffected by any treatment E: Liver Mogat1 gene expression was reduced by Mogat1 ASO treatment compared to controls. F-I: Liver gene expression markers of interferon signaling are increased in Mogat1 ASO treated mice and inhibited by INFR-Ab. J: Epididymal Mogat1 gene expression was decreased by Mogat1 ASO treatment. K-N: Epididymal gene expression markers of interferon signaling are inhibited by INFR-Ab. O-Q: Expression of adipose beiging genes were increased by Mogat1 ASO and inhibited by INFR-Ab treatment. Data are expressed as means \pm S.E.M. \# $p<0.05$ from Scramble ASO $+p<0.05$ from IgG controls; $n=5$. 